\title{
PATTERN-RECONFIGURABLE ANTENNA FOR 5.5-GHZ WIMAX APPLICATIONS
}

\author{
FAYYADH H. AHMED \\ Dept. of Electrical and Computer, University of Duhok, Kurdistan Region- Iraq
}

\begin{abstract}
Pattern reconfigurable antenna, which is based on multi-switchable radiators with rectangular reflectors, is presented. The antenna utilized the self-similarity double ring configuration property in the form of rectangular and a Wilkinson power divider feeder, to exhibit wideband and radiation pattern reconfiguration characteristics. The pattern reconfiguration is achieved by feeding these self-similarity double rings of antenna in two directions. A few switches and power divider is used in order to change the feeding direction.

The antenna is capable of steering the beam in the four directions $25^{\circ}, 52^{\circ}, 155^{\circ}$ and $158^{\circ}$ in the azimuth plane and, four directions $20^{\circ}, 10^{\circ}, 5^{\circ}$, and $51^{\circ}$ in the elevation plane, depending on the state of switches loaded on the rectangular rings. Initial results were obtained by using short and open circuits to represent ON and OFF for switches respectively.

The proposed reconfigurable antenna has the advantage of miniaturization, simple reconfigurable, low profile, light weight, and easy fabrication.

For verification of simulation results, the proposed antenna is fabricated and the prototype is measured using R\&S ZVL 13 Vector Network Analyzer. The results show a good agreement between simulated and measured results.
\end{abstract}

KEYWORDS: Pattern reconfigurable, Switch, self-similarity, Wilkinson power divider, WiMAX.

\section{INTRODUCTION}

$\mathbf{R}$ ecently, the antennas in modern applications have to be multi-functional or adaptable with the system/user requirements. Fixed-performance antennas are limited in their capability to cope with the changing scenario; thus, reconfigurable antennas have become the cost-effective solution to that situation. Moreover, reconfigurable antennas can save space and provide flexibility by replacing several fixed antennas to meet the system's requirements [1].

In particular, radiation pattern reconfiguration characteristic at a fixed operation frequency has received much attention because it can be used to avoid noise source, improve security and save energy by better directing signal toward intended users [2].

The phase controlled antenna array is a traditional technique of beam steering, though the constraints like feeding complexity, size, weight etc. and overall cost limited them to few applications. Considering these, planar reconfigurable radiation pattern antennas, have been widely investigated because of their attractive features such as simple structure, low profile, light weight and ease of fabrication and integration [1].

To design a reconfigurable beam pattern antenna effectively is a challenge to the researcher since there is still no universal approach to design it. Several designs in the literature have targeted pattern reconfigurable antennas. Some of these designs are very limited as they can only switch their beams in few directions [3].

Some others based on an antenna with multifeed mechanisms [4], [5], and [6], and the pattern switching is realized by selecting one of the antenna feeds as the signal input port.

The other method is based on a single-feed antenna, and the antenna operation mode can be varied through controlling the states of the PIN diodes which need to be properly placed in the antenna structure [3] and [7].

In this paper, a pattern reconfigurable antenna based on self-similarity double rectangular ring configuration and single-feed probe antenna is proposed. A suggested antenna is operates around 5.5 GHz for WiMAX application. The proposed 
antenna has two self-similarity double rectangular rings acts as director, placed around rectangular patches acts as reflector. The self-similarity rings are placed $45^{\circ}$ with respect to each other. A single microstrip feeding line with Wilkinson power divider is used to feed the antenna. Pattern reconfiguration is accomplished by switching the feeding from a Wilkinson power divider to both self-similarity rectangular double rings alternatively in two directions.

\section{ANTENNA CONFIGURATION AND RADIATION RECONFIGURATION MECHANISM}

\section{Antenna Design:}

The final layout of the proposed antenna, along with its dimensions is presented in Fig. 1a, and its dimensions are illustrated in Table 1.Initially, the structure is inspired from self-similarity property of the fractal geometry. To implement the selfsimilarity principle each antenna is formed of two similar in-shape rings, where the inner ring is a scaled copy of the outer one. The dimensions of the two rings were initially chosen such that the mean circumference of each ring corresponds to the effective wavelength at the center of the wanted frequency band. Thus, the outer ring corresponds to the lower band while the inner one corresponds to the upper band. The dimensions can then be slightly changed or optimized to get better response using a software package [8].

The antenna is designed to operates at 5.5 GHz. Antenna's side $\left(\mathrm{W}_{1}\right)$ is selected to be =20_mm (multiples of $\left.\lambda_{\text {eff }} / 4\right)$ as stated by the equation [9]:

$L_{p}=\frac{\lambda_{\text {eff }}}{4}=\frac{\lambda}{4 k}=\frac{c}{4 k f_{L}}$

Where, $\lambda$ and $c$ is the free space wavelength and speed of the light, respectively; $\lambda_{\text {eff }}$ is effective wavelength and is given by [10]:

$\lambda_{\text {eff }}=\frac{\lambda}{\sqrt{\varepsilon_{\text {reff }}}}$
The approximated value of $\varepsilon_{\text {reff }}$ is given by [9]:

$$
\varepsilon_{\text {reff }} \approx \frac{\varepsilon_{r}+1}{2}
$$

Double rectangular rings have been fed from the output ports of a Wilkinson power divider via switches and ensuring a match to $50 \Omega$.

The Wilkinson power divider has been used because it is easy to construct and has some extremely useful properties like; matched at all ports, large isolation between output ports, reciprocal, and lossless when output ports are matched [11]. The Wilkinson power divider is considered to be the equal-split $(3 \mathrm{~dB})$ case. This divider is often made in microstrip line or stripline form, as depicted in Fig.1b.

Therefore to achieve characteristic impedance $\left(Z_{0}\right)$ of $50 \Omega$, the microstrip line width has been calculated using the equation [9]:

$$
Z_{0}=\frac{87}{\sqrt{\varepsilon_{r}+1.41}} \ln \left(\frac{5.98 h}{0.8 W_{\text {strl }}+t}\right)
$$

Where $h$ is the substrate's thickness of 1.6 _mm, $\quad t$ is the metallization thickness of $0.035 \_\mathrm{mm}, W_{\text {strl }}$ is the microstrip line width, and $\varepsilon_{r}$ for the FR4 substrate is 4.3. Therefore, according to (7), and for a characteristic impedance of $50 \Omega, W_{\text {strl }}$ must be set equal to 3_mm, and the impedance for $\lambda / 4$ transformer should be $70.7 \Omega\left(\sqrt{2} Z_{0}\right)$, thus its microstrip width must be equal to 1.672 mm.

A good impedance matching was observed at the $5.5 \mathrm{GHz}$ for the structure shown in Fig.1. The two patches (collective double rectangular rings with rectangular reflectors) are separated from a Wilkinson output ports by $0.5 \times 3$ mm gaps. Two switches (SW1 and SW2) are used to activate one by one between patch 1 and 2 . Also, the rings were separated from feeding by inserting $0.5 \times 2$ mm gap between feed line and rings, four collective switches (SW3, SW4, SW5 and SW6) are used in the gaps between the feedline and the rings to excite the rings alternatively in both directions (left and right). 


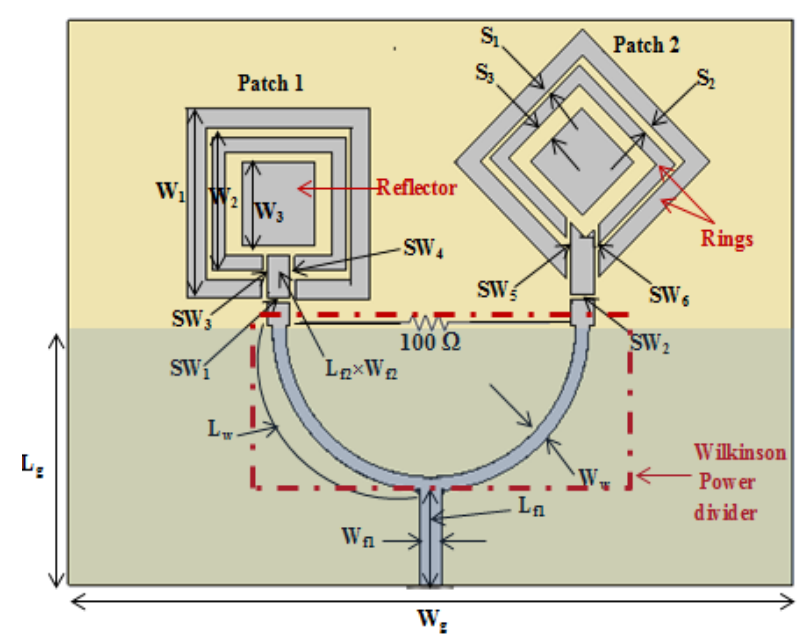

(a)

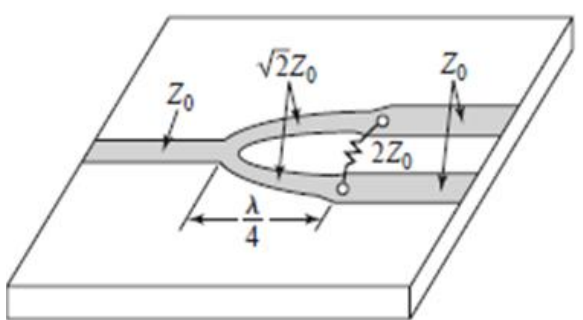

(b)

Fig (1):-- (a) Structure of the proposed antenna (b) An equal-split Wilkinson power divider in microstrip line form.

Table( 1):-Optimum dimension of the antenna depicted in Fig. 1

\begin{tabular}{|c|c|c|c|c|c|c|}
\hline Antenna's parameter & $\mathbf{W}_{1}$ & $\mathbf{W}_{2}$ & $\mathbf{W}_{3}$ & $\mathbf{S}_{1}$ & $\mathbf{S}_{2}$ & $\mathbf{S}_{3}$ \\
\hline Dimension in (mm) & 20 & 14 & 9 & 0.62 & 1 & 1.87 \\
\hline Antenna's parameter & $\mathbf{L}_{\mathbf{f 1}}$ & $\mathbf{W}_{\mathrm{f} 1}$ & $\mathbf{L}_{\mathbf{f} 2}$ & $\mathbf{W}_{\mathrm{f} 2}$ & $\mathbf{L}_{\mathrm{W}}$ & $\mathbf{W}_{\mathbf{W}}$ \\
\hline Dimension in (mm) & 10 & 3 & 4.5 & 3 & 24 & 1.67 \\
\hline Antenna's parameter & $\mathbf{L}_{\mathrm{g}}$ & $W_{g}$ & \multicolumn{4}{|c|}{ Total Volume } \\
\hline Dimension in (mm) & 30 & 80 & \multicolumn{4}{|c|}{$60 \times 80 \times 1.6 \mathrm{~mm}^{3}$} \\
\hline
\end{tabular}

\section{PATTERN RECONFIGURABLE MECHANISM}

The greatest challenges faced by antenna designer in pattern reconfigurable antenna design is how it can be reconfigure far field parameters, while remain input impedance parameters unchanged. This can be done when antenna structure remain fixed.

Reconfiguration can be obtained electrically by somehow redistributing surface current distribution and thus changing the electromagnetic fields of the antenna [12].

In this paper, a new idea is suggested to redistributing the current on the antenna structure while remaining it fixed. Two patches are excited by two ports of a Wilkinson power divider alternatively using SW1 and SW2 as shown in Fig 1.

The patches are placed $45^{\circ}$ with respect to each other. The first self-similarity double ring configuration gets excitation from output port 1 of a Wilkinson power divider through two directions (left and right), via switches SW3 and SW4 as shown in Fig 1., also the second one gets the feeding also from both sides through two other switches SW 5 and SW6.

However, when SW1 is ON and SW2 OFF, patch1 operates as director and patch 2 as reflector. In addition, when switch 3 is $\mathrm{ON}$ and switch 4 OFF (state 1), the first self-similarity double ring of the patch 1 is connected to the feed line (port1) from left side, therefore the current flows through patch 1 from left side providing clockwise current distribution direction, and hence providing particular radiation pattern. While when SW3 OFF and SW4 ON (state2), patch 1 is excited from the right side, and thereby gives another current distribution form (counter clockwise) on the antenna structure, subsequently, another radiation pattern is configured. In this 
method, since in both states the physical dimensions for the antenna is not altered, and the switches are symmetric with respect to the antenna's feeding structure, thus the frequency response of reflection coefficient for these states are almost same at 5.5 resonant frequency.

In like manner, when SW1 OFF and SW2 ON the patch 2 acts as director while patch 1 acts as reflector. Moreover, when SW5 ON and SW6 OFF (state 3), the current is injected to patch 2

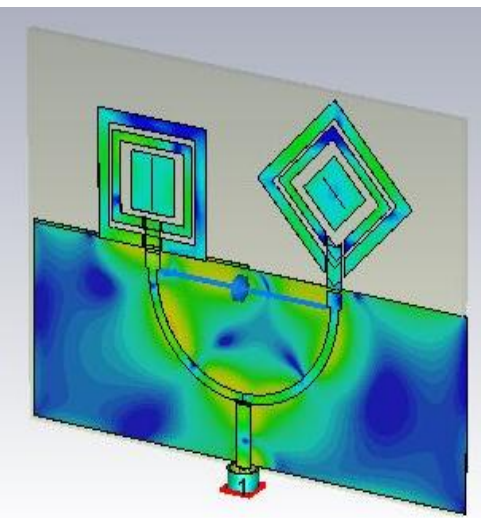

(a)

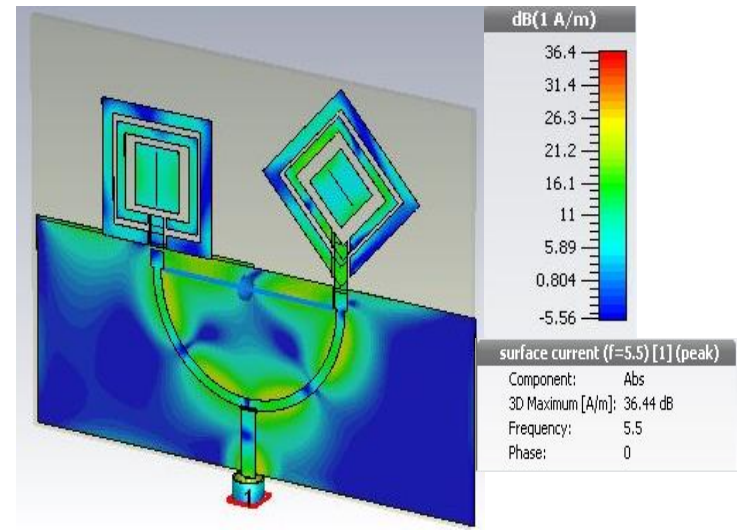

(c)

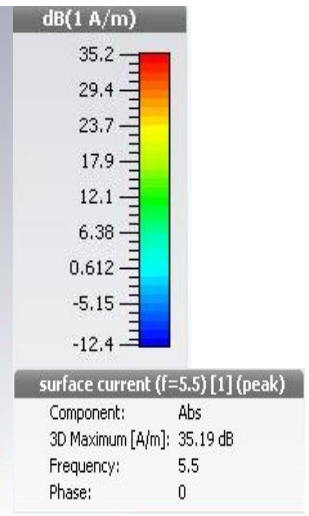

$\mathrm{dB}(1 \mathrm{~A}$

$26.3=$

11

0.804

$-5.56$ from left direction, and as long as the patch 2 has identical dimensions to patch 1 but rotated by $45^{\circ}$, so it has the same input impedance of patch 1 at 5.5 resonant frequency, while the current distribution will has different scheme with respect to states 1 and 2, thus, provides third pattern form. At last, if SW5 OFF and SW6 ON (state 4) the current is supplied to the patch 2 from right side, therefore fourth dissimilar current distribution is obtained as shown in Fig. 2.

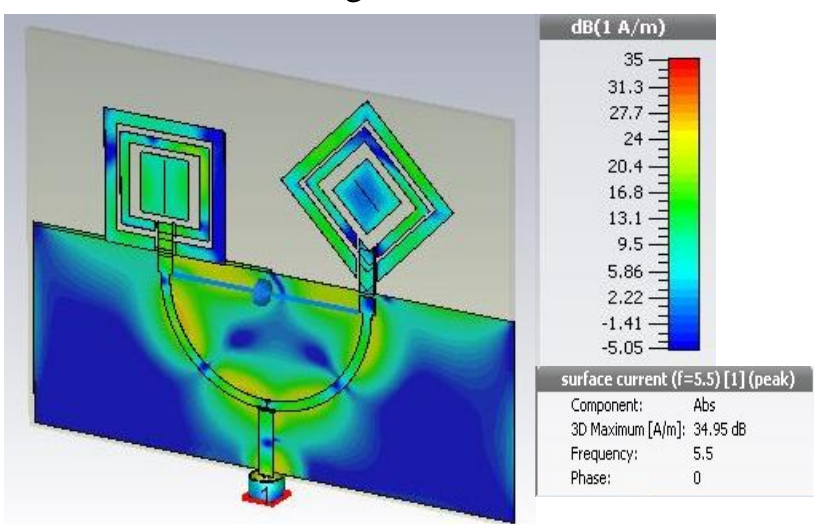

(b)

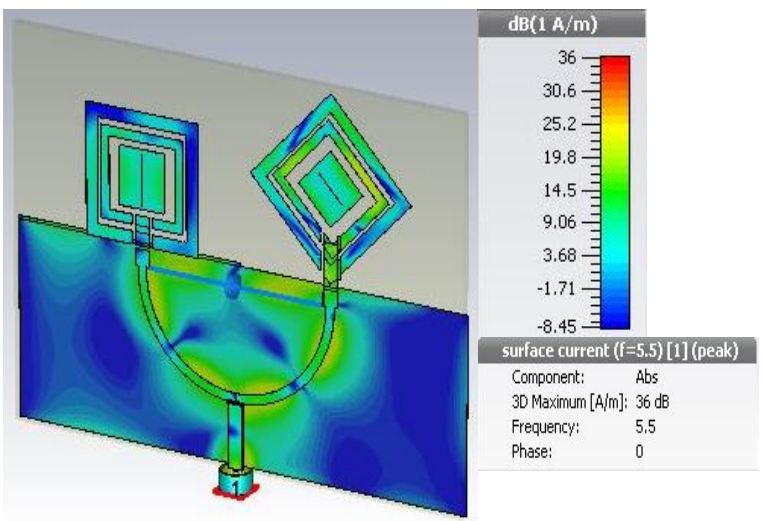

(d)

Fig. (2):- Simulated CST software's surface current density distributions for the antenna in Fig.1for; (a) State 1, (b) State2(c) State 3, and (d) State 4

\section{SIMULATION AND MEASURED RESULTS}

The performance of the designed antenna were simulated and analyzed by CST 2014 Microwave Studio in terms of return loss measurement, gain, efficiency, and radiation pattern. Meanwhile, the proposed antenna has been fabricated as in Fig.3a, and the experimental measurements are conducted in terms of return loss and radiation pattern using
Rohde \& Schwarz ZVA I3 vector network analyzer (VNA) (refer to Fig. 3b). 


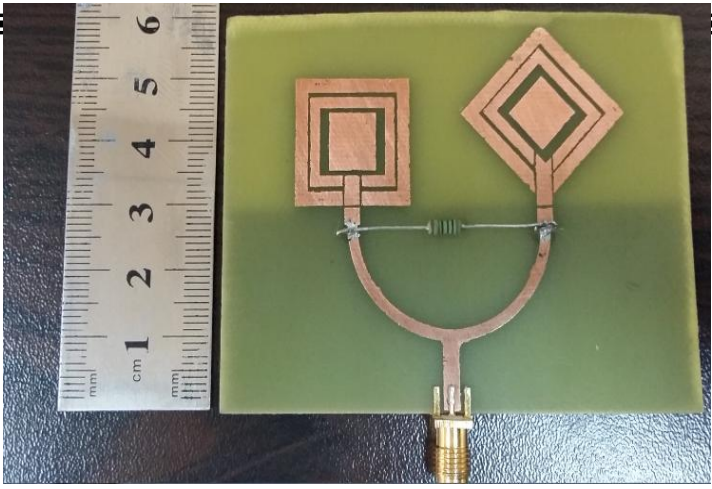

(a)

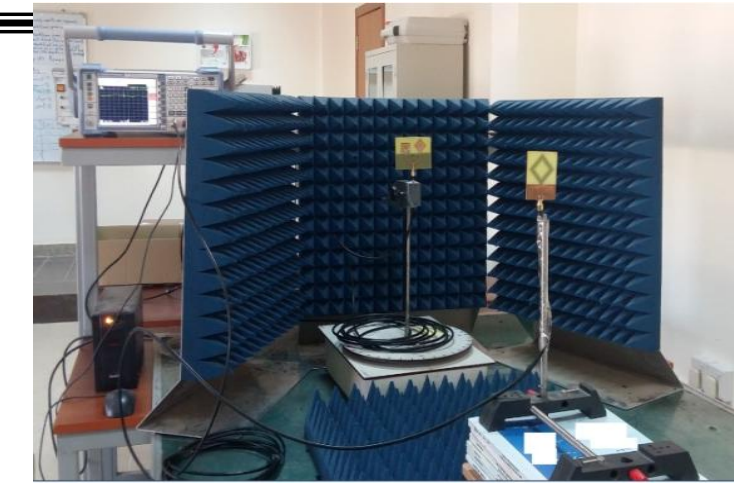

(b)

Fig.3:- (a) Prototype of the antenna, (b) Radiation pattern measurement of the proposed antenna

\section{Reflection coefficient:}

The investigation of various rings arrangements incorporated with the switches provided a feasibility to design pattern reconfigurable planar antenna. The antenna was designed and optimized to operate at various radiation configurations for different switch conditions, while works at single $5.5 \mathrm{GHz}$ WiMAX frequency. The RF switches used in simulations are realized as metal pads with dimensions $0.5 \mathrm{~mm} \times 2 \mathrm{~mm}$, while a point of solder in practical work. Fig. 4 shows the comparison between simulated and measured input reflection coefficient curve for the antenna for different switches states.

A little discrepancy between the simulated and measured results may be attributes to the cable

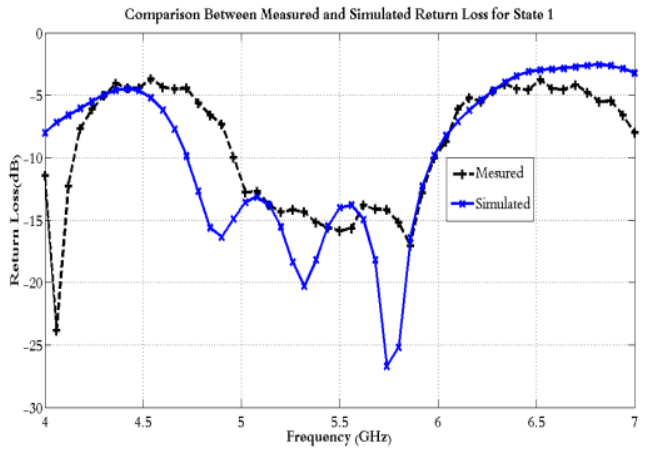

(a) and connector was used in measurements, where they were not considered in simulation. Also, the antenna's manufacturing tolerance may be having an effect on measured results. However, in the simulation the switch is modeled using metal pads, while in the fabrication process, a lump solder is used to short circuit the switch in case of ON state, and in OFF states as open circuit.

The simulated $-10 \mathrm{~dB}$ return loss bandwidth around 5.5 resonant frequency, is slightly different in first two states from second two states, but at desired resonant frequency $(5.5 \mathrm{GHz})$, they are get good match, and their frequency bandwidths are $1.25 \mathrm{GHz}, 1.02 \mathrm{GHz}, 0.559 \mathrm{GHz}$, and $0.458 \mathrm{GHz}$, respectively, the corresponding $-10 \mathrm{~dB}$ bandwidth is suitable for IEEE 802.11a $5.5 \mathrm{GHz}$ WiMAX band.

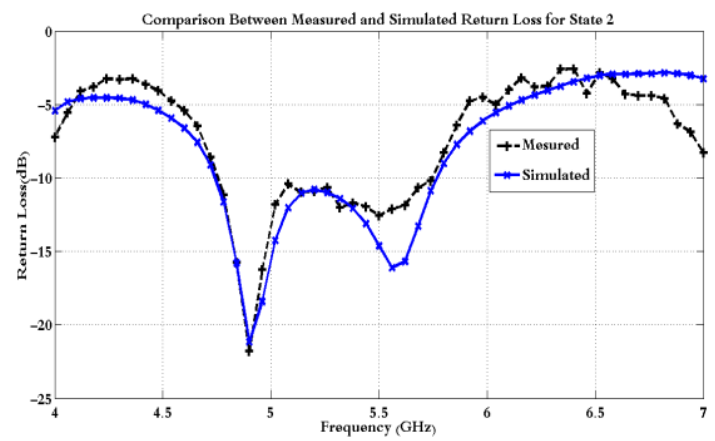

(b) 


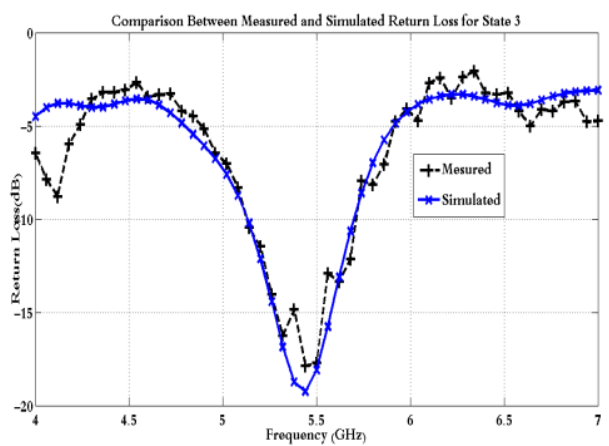

(c)

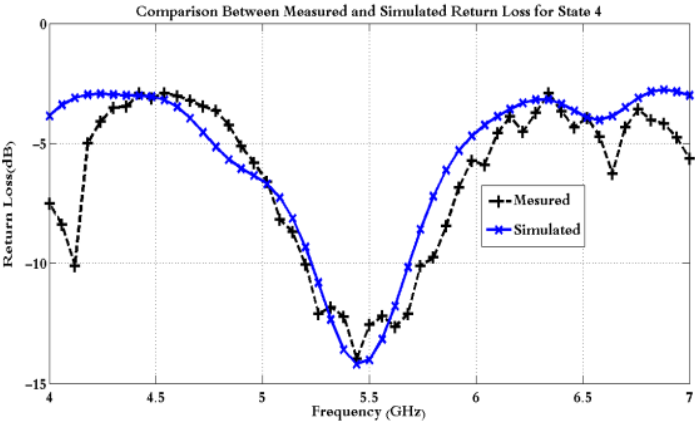

(d)

parison between simulated and measured reflection coefficient of proposed antenna for different switch configurations.

\section{Radiation Pattern Performance:}

The measured and simulated radiation patterns of both the Azimuth (xy- plane) and Elevation (xz-plane) planes are plotted in Fig. 5. It can be seen, for states $1,2,3$, and 4 the maximum radiation in azimuth planes are $25^{\circ}, 52^{\circ}, 155^{\circ}$, and $158^{\circ}$, respectively, and in elevation planes are $21^{\circ}, 10^{\circ}, 5^{\circ}$, and $51^{\circ}$ respectively.

In Fig. 6, the simulated 3D radiation patterns are also plotted. It can be seen in Fig. 6, that the patterns have a different maximum gain in both azimuth and elevation planes for various switches states.
Fig.7 shows the radiation pattern characteristic for the patches of the antenna. From the figure it is clearly found that less than -15 $\mathrm{dB}$ of cross-polarization is obtained for both, patch 1 and patch 2 . This result indicates that polarization stability for the proposed antenna is realized properly.

The summary for various switches states results in terms of operating resonant frequency, maximum radiation direction in azimuth and elevation planes, HPBW, gain, and antenna efficiency are displayed in Table 2

Table( 2):- The Summary for various switches cases

\begin{tabular}{|c|c|c|c|c|c|c|c|}
\hline \multirow[t]{2}{*}{ State } & \multirow[t]{2}{*}{ Bandwidth } & \multicolumn{2}{|c|}{$\begin{array}{c}\text { Maximum Radiation } \\
\text { Direction }\end{array}$} & \multicolumn{2}{|c|}{ HPBW } & \multirow[t]{2}{*}{$\begin{array}{l}\text { Simulated } \\
\text { Gain(dBi) } \\
\end{array}$} & \multirow[t]{2}{*}{$\begin{array}{c}\text { Antenna } \\
\text { Effeciency }\end{array}$} \\
\hline & & Elevation & Azimuth & $\begin{array}{l}\text { Elevatio } \\
\mathrm{n}\end{array}$ & Azimuth & & \\
\hline 1 & $1.25 \_\mathrm{GHz}$ & $21^{\circ}$ & $25^{\circ}$ & $41.7^{\circ}$ & $30.9^{\circ}$ & 3.42 & $56.2 \%$ \\
\hline 2 & 1.02_GHz & $10^{\circ}$ & $52^{\circ}$ & $69.3^{\circ}$ & $38.3^{\circ}$ & 4.27 & $58.3 \%$, \\
\hline 3 & 559_MHz & $5^{\circ}$ & $155^{\circ}$ & $132.1^{0}$ & $40.8^{\circ}$ & 5.71 & $66.7 \%$, \\
\hline 4 & 458.8_MHz & $51^{\circ}$ & $158^{0}$ & $67.1^{\circ}$ & $38.5^{\circ}$ & 4.24 & $65.4 \%$ \\
\hline
\end{tabular}



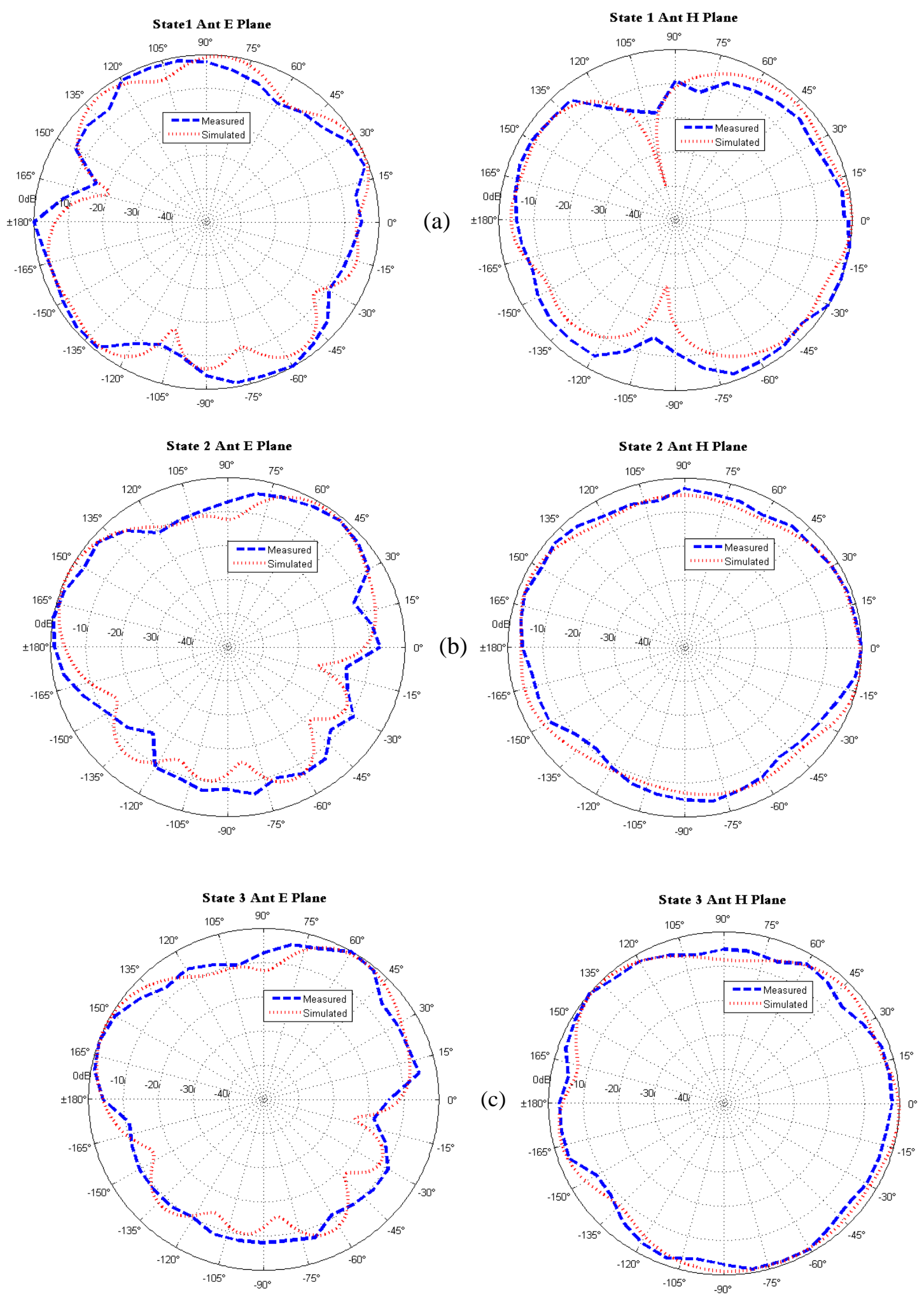

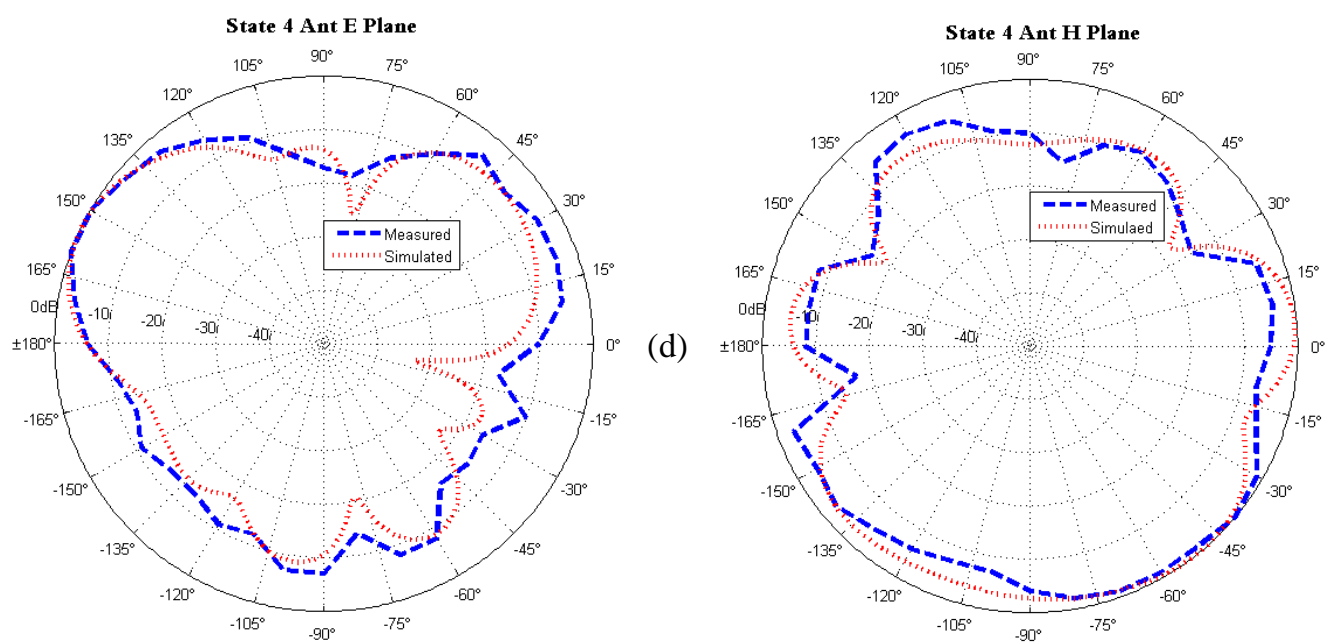

Fig (5):- Simulated and measured radiation pattern of proposed antenna.at (a) state1, (b) state2, (c) state 3, and (d) state4; left column x-y_plane, right column x-z_plane.

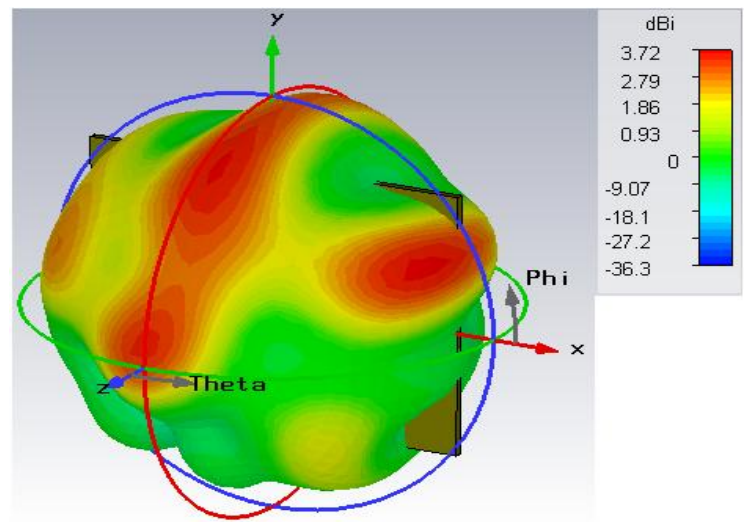

(a)

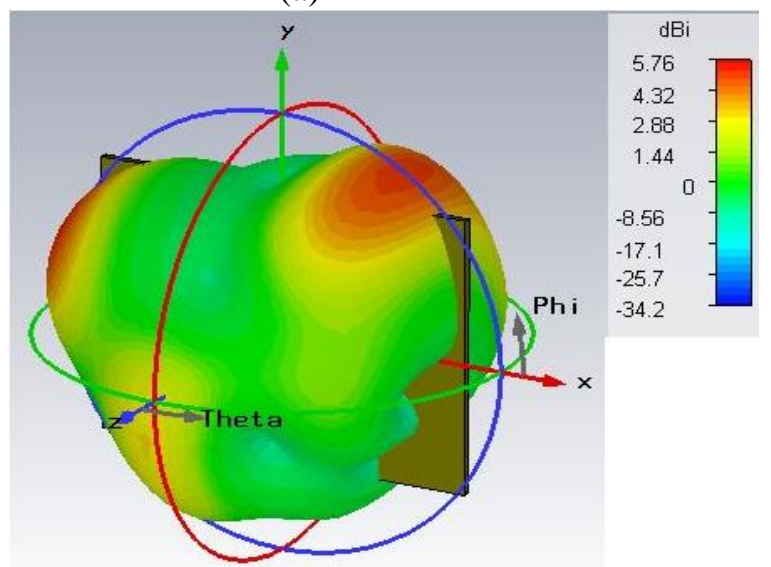

(c)

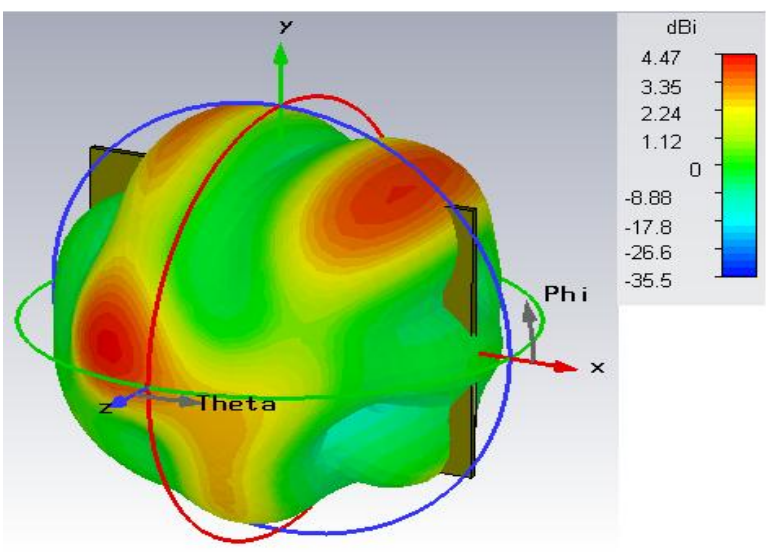

(b)

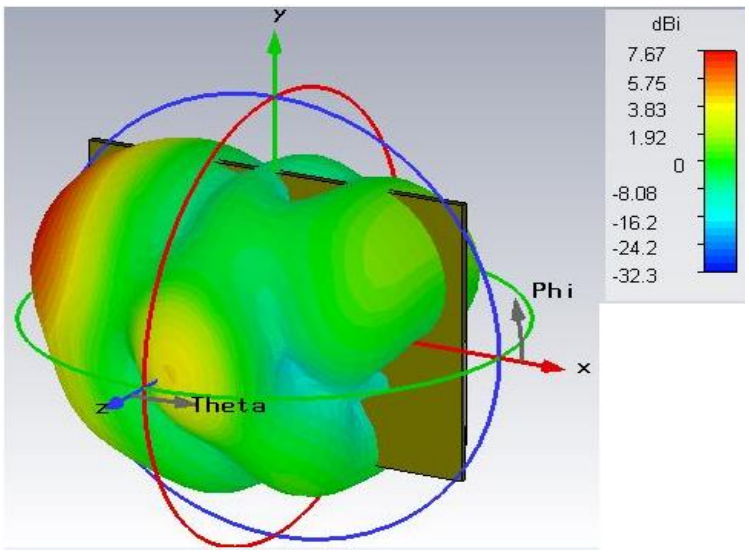

(d)

Fig (6: 3):-D radiation pattern of proposed antenna.at (a) state1, (b) state2, (c) state 3, and (d) state4. 


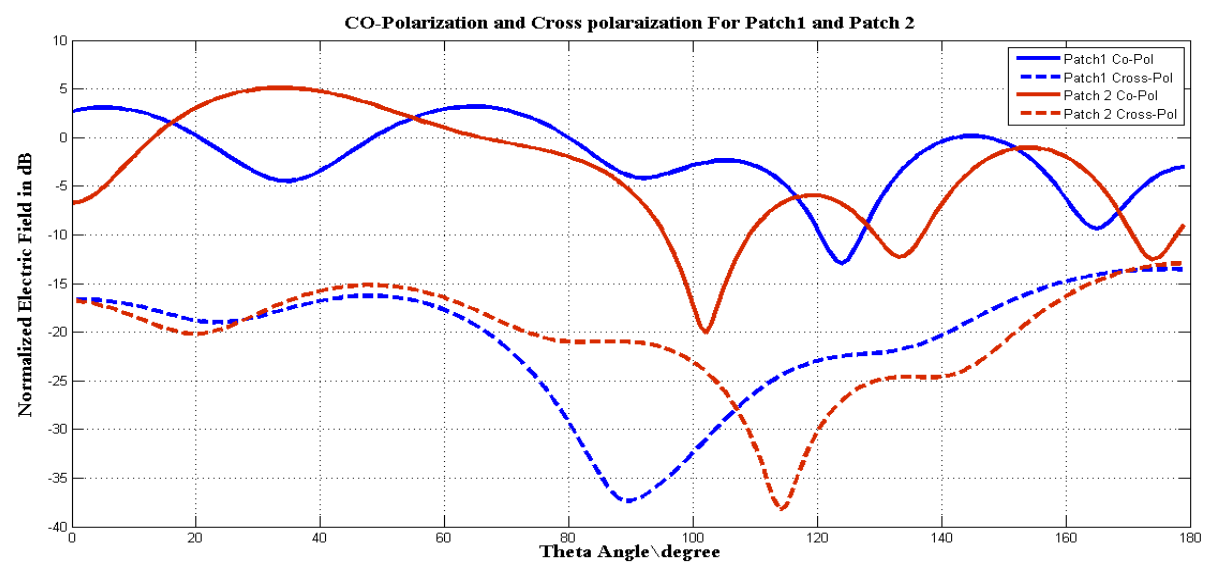

Fig (7):-: Co- and Cross- Polarization for the proposed antenna Patch 1 and Patch 2

\section{CONCLUSION}

A pattern reconfigurable antenna at 5.5 WiMAX band is presented. The antenna is based on multi-switchable radiators. Two self-similarity double ring configurations placed $45^{\circ}$ with respect to each other. Several switches with a Wilkinson power divider are used to connect/disconnect the rings to feed line, and consequently to change the surface current on the antenna structure. A prototype was fabricated and tested experimentally, and measured results are in good with the simulated. Four different patterns with realized gain level greater than $3.4 \mathrm{dBi}$, in elevation and azimuth are successfully obtained at $5.5 \mathrm{GHz}$. Hence, the suggested antenna is a good candidate for WiMAX applications.

\section{REFERENCES}

[1] M.S. Alam and A.M. Abbosh, " Wideband Pattern Reconfigurable Antenna Using Pair of Radial Radiators on Truncated Groundwith Switchable Director and Reflector", IEEE Antennas and Wireless Propagation Letters, December, 2016.

[2] Imen BEN TRAD, Jean Marie FLOC'H, and Fethi CHOUBANI, "Design of Reconfigurable Radiation Pattern Ring- Dipole Antenna for Wireless Communication", 7th European Conference on Antennas and Propagation (EuCAP), 2013.

[3] Tamer Aboufoul, Imran Shoaib, Akram Alomainy, and Xiaodong Chen, "Pattern Reconfigurable Planar UWB Antenna Array for Future Cognitive Radio Portable Devices", Loughborough Antennas \& Propagation Conference, 12-13 November 2012.

[4] $\mathrm{Na}$ Kou and Long Li, "Frequency and Pattern Reconfigurable Annular Slot Antenna with Two Feeding Ports", Wireless Symposium (IWS), 2014 IEEE International, 24-26 March 2014.
[5] Yan-Ying Bai, Shaoqiu Xiao, Changrong Liu, Xiang Shuai, and Bing-Zhong Wang, "Design of Pattern Reconfigurable Antennas Based on a Two Element Dipole Array Model", IEEE Transactions On Antennas and Propagation, Vol. 61, No. 9, September 2013.

[6] Amit Mehta and Shivam Gautam ," Planar Pattern Reconfigurable Antenna Integrated

With a WiFi System for Multipath Mitigation and Sustained High Definition Video Networking in a Complex EM Environment", IEEE International Symposium on Antennas and Propagation \& USNC/URSI National Radio Science Meeting, 2015.

[7] M. Saeed Khan, Sajid M. Asif, and Raed M. Shubair,“ A Pattern Reconfigurable Printed Patch Antenna", IEEE International Symposium on Antennas and Propagation (APSURSI), 2016.

[8] KH. H. Sayidmarie, T.A.Nagem, "Compact DualBand Dual-Ring Printed Monopole Antennas For WLAN Applications", Progress In Electromagnetics Research B, Vol, 43, PP.313$331,2012$.

[9] Y. A. Fadhel, KH. H. Sayidmarie, " Design and implemtation of ultra wideband antenna for imaging application", Ph.D thesis, University of Mosul, Iraq, July, 2013.

[10] A. Foudazi, H. Hassani, and S. Nezhad, "Small UWB Planar Monopole Antenna with Added GPS/GSM/WLAN Bands," IEEE Trans. on Antennas and Propag., vol. 60, no. 6, jun. 2012.

[11] David M. Pozar, Microwave Engineering, JohnWiley \& Sons, Inc, 4th ed., 2011.

[12] Meraline Selvaraj and B. S. Sreeja, "Pattern Reconfigurable Antenna for "Wireless Applications using Parasitic Ring," IEEE WiSPNET 2016. 
